\title{
ARITICLES ORIGINAUX
}

\section{Antigène lipopolyosidique de Pasteurella multocida type $\mathrm{E}$}

\author{
par P. PERREAU ef J.-P. PETIT \\ avec lo collaboration technique de $M^{\text {me }}$ D. BERGERON, Mlle P. GAYT ef de Mme A. MARQUET
}

L'existence d'un type sérologique de Pasteurella multocida, agent de la pasteurellose septicémique des bovins africains et distinct des quatre types classiques connus jusqu'ici, vient d'être établi récemment $(4,11)$.

La septicémie pasteurellique observée sur le bétail d'Afrique centrale ef occidentale ne se distingue de la septicémie classique due au type $B$, observée surtout en Asie, par aucun signe particulier tant du point de vue clinique que nécropsique.

Nous avons déjà montré que la distinction antigénique peut être faite entre les souches de type $B$ et les souches africaines au moyen de trois méthodes : hémagglutination passive, séro-protection de la souris et précipitation en gélose (11).

Nous avions utilisé dans ces tests des antigènes bruts, préparés à partir de bactéries entières traitées par la chaleur, les ultra-sons, ou des alternances de congélation et de décongélation.

Désirant approfondir et confirmer la spécificité sérologique du type africain, nous avons isolé les antigènes lipopolyosidiques des souches d'Afrique centrale afin de les comparer à ceux des souches du type $B$.

L'étude des lipopolyosides de Pasteurella multocida a déjà fait l'objet de divers travaux, et certains sont anciens, qui méritent d'être rappelés ici.

PIROSKY $(12,13,14)$ en 1938, utilisant la méthode de BOIVIN et MESROBEANU, avait isolé un complexe glucido-lipidıque auquel il avait reconnu une spécificité sérologique ainsi que des propriétés toxiques et immunisantes.

Rev. Elev. Méd. vét, Pays. trop. 1963, 16, n0 1.

Reçu pour publication : avril 1963.
CARTER et ANNAU (5) en 1953 ont préparé des polyosides capsulaires à haute spécificité sérologique, qu'ils ont distingués de l'« antigène de Boivın », ce qui était sans doute inexact, car, à la lumière des données actuelles, leurs extraits devaient contenir aussi des lipopolyosides de surface.

MAC LENNAN et RONDLE (9) en 1957 ont extrait par le phénol des lipopolyosides pyrogènes dont la spécificité sérologique était mise très nettement en évidence par des tests de précipitation en gélose utilisant des sérums absorbés par des antigènes communs. Etant donné que cette spécificité était exactement parallèle à la spécificité immunologique révélée par les épreuves de séroprotection des souris, ils pensaient que ces antigènes devaient être protecteurs, sans pouvoir l'affirmer d'ailleurs car d'autres antigènes spécifiques et protecteurs pouvaient exister dans la bactérie.

BAIN et KNOX (2) en 1961 font une étude détaillée des antigènes giucido-lipidiques de Pasteurello multocida type 1 (souche INSEIN), montrent leur toxicité et leur rôle important dans le phénomène d'hémagglútınation passive et analysent leur constitution chimique par chromatographre.

La présente étude poursuit le même but que ce dernier travail en s'adressant à un type sérologique distinct, bien que voisin, de Pasteurella multocida ; nous pensons, en effet, qu'il est d'un grand intérêt d'assurer sur le plan de la chimie biologique ef de l'immunologie la différence qui peut exister entre les deux types bactériens qui sont responsables de la septicémie hémorragique des bovins. 


\section{MATÉRIEL ET MÉTHODES}

\section{Souches de «Pasteurella multocida 》:}

Les souches de type $E$ sont les souches P7, P11 et $\mathrm{P} 13$, isolées en Adamaoua (Cameroun). La dernière a été récoltée en septembre 1962 et a servi à la production de lipopolyoside spécifique dès son isolement.

Comme souches de type $B$, nous avons employé pour comparaison, la souche Insein de Birmanie, et secondairement la souche R2 d'Iran.

\section{Préparation des antigènes lipopolyosidiques :}

10 Objention des suspensions boctériennes.

Des suspensions denses de Pasteurella mufocido sont récoltées dans un appareil à culture aérée et agitée par tourbillon (10); le milieu est celui de STERNE et l'antimousse est le Rhodorsit 426 Rhône-Poulenc.

Les récoltes sont centrigugées à $15.000 \mathrm{t} . / \mathrm{mn}$ dans une centrifugeuse Servall à dispositif de débi continu (SZENT-GORGYI). Cette centrifugation permet l'élimination totale de l'antimousse ef l'obtention de culots microbiens très essorés.

Les germes sont repris par un faible volume d'eau distillée, de façon à obtenir une suspension très dense : en moyenne, $20 \mathrm{~g}$ de bactéries sèches pour un litre d'eau.

Cette suspension est homogénéisée soigneusement d̀ l'aide d'un apparell «Ultra-Turrax» TP 18/2.

\section{$2^{\circ}$ Préparation des lipopolyosides.}

La méthode employée est celle deWESTPHAL $(18,19)$; la suspension est traitée par un égal volume d'une solution de phénol à 90 p. 100 à chaud (une demi-heure à $65-68^{\circ} \mathrm{C}$ ), I'homogénéisation du mélange phénol-suspension étant effectuée aussi à l'Ultra-Turrax.

Ce mélange, refroidi immédiatement, est conservé une nuit à 40 ; la couche aqueuse de surface, décantée, subit deux centrifugations étagées pour être débarrassée de tout élément figuré.

Cette phase aqueuse est mise en dialyse continue ( 3 à 4 jours) jusqu'à ce qu'on ne puisse plus $y$ déceler de phénol ; elle est ensuite concentrée ef lyophilisée. On obtient alors un produit brut, riche en acide nucléique, que l'on peut conserver longfemps sans altérations ou purifier immédiatement.

Cette purification est faite d'abord par précipitation à l'alcool à $95^{\circ}$ (15 p. 100 en volume), après remise de l'antigène en solution dans de l'eau distillée. Deux précipitations successives sont effectuées; les culots sont rejetés et le surnageant seul est conservé. C'est un liquide à opalescence bleu-jaune, légèrement visqueux et qui ne mouille pas'le verre.

La dernière purification est faite par centrifufugation : après 4 heures à $37.000 \mathrm{~g}$ (centrifugeuse Servall RC-2, Rotor SS 34), le lipopolyoside apparaît au fond des pots sous forme d'un disque blanc et visqueux. Ce sédiment, après rejet du surnageant, est remis en solution dans de l'eau distillée et centrifugé une seconde fois dans des conditions identiques.

Le lipopolyoside purifié obtenu est lyophilisé.

\section{Méthodes de dosage:}

Ces échantillons lyophilisés ont servi à préparer des solutions aqueuses contenant toutes $1,0 \mathrm{mg} / \mathrm{ml}$ du produit isolé.

Sur ces solutions, l'azote a été dosé selon la méthode de KJELDAHL après minéralisation sulfurique et oxydante $\left(\mathrm{H}_{2} \mathrm{O}_{2}\right)$, le phosphore acidosoluble total par la technique spectrophotométrique de DELSAL et MANHOURI (7) modifiée pour permettre le dosage de $0,5 \mu \mathrm{g}$ dans $10 \mathrm{ml}$ en effectucint la lecture d̀ $830 \mathrm{~m} \mu$.

Les protéines ont été déterminées par la méthode du biuret selon ARDRY (1), mais cette excellente méthode n'était pas assez précise pour les très faibles quantités que nous avions à doser. Aussi l'avons nous complétée par l'établissement du spectre ultraviolet de 230 d̀ $390 \mathrm{~mm}$ et par la défermination des rapports des coefficients d'extinction: $\frac{K 280}{K 310}$ ef $\frac{K 280}{K 252}$; la solution témoin était un sérum de bovin dilué contenant 3,373 mg de protéines par $\mathrm{ml}$.

De la même façon les dosages d'acide rıbonucléique à $260 \mathrm{m \mu}$ ont été complétés par la détermination des rapports des coefficients d'extinction $\frac{\mathrm{K} 260}{\mathrm{~K} 310}$ ef $\frac{\mathrm{K} 260}{\mathrm{~K} 230}$; le témoin était une solution d'acide ribonucléique Fluka « purum». Toutes ces déterminations et ces dosages 
spectrophotométriques ont été faits avec un appareil Zeiss PMQ-II, les mesures de $\mathrm{pH}$ avec le potentiomètre Metrohm E 353.

Les concentrations sous vide intervenant après chaque précipitation alcoolique ont été faites sans que la solution traitée dépasse la température de $60^{\circ} \mathrm{C}$ dans un Rotovopor. Buchi.

\section{Méthodes sérologiques:}

L'hémagglutination indirecte est faite selon la méthode de CARTER (3) en utılisant soit des hématies $O$ humaines fraîches, solt des hématies de mouton fraîches ou formolées, en suspension à 1 p. 100 et sensibilisées par des solutions de concentrations diverses en lipopolyoside ; elle est effectuée en tubes et non sur plaque.

La précipitation en gélose utilise le milieu suivant ajusté à $\mathrm{pH} 7$.

$\begin{array}{lr}\text { gélose noble Difco } \ldots \ldots \ldots & 12 \mathrm{~g} \\ \text { chlorure de sodium } \ldots \ldots & 8,5 \mathrm{~g} \\ \text { merthiolate de } \mathrm{Na} \ldots \ldots \ldots & 0,1 \mathrm{~g} \\ \text { equ distillée } \ldots \ldots \ldots \ldots & 1.000 \mathrm{ml}\end{array}$

L'immunoélectrophorèse a été pratiquée en gélose noble Difco à 1 p. 100, tamponnée au

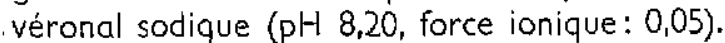
selon la méthode de GRABAR et WILLIAM (8), mais sur des lames porte-objet (microanalyse de SCHEIDEGGER (15); Le mélange tampon-gélose (à parties égales) est étalé sur les lames en couche uniforme de $3 \mathrm{~mm}$ d'épaisseur ; celles-ci sont soumises ensuite, dans une cuve (Chaix) permettant une évaporation contrôlée, à une tension de $9.4 \mathrm{~V} / \mathrm{cm}$ pendant $50 \mathrm{mn}$.

Les protéines sont alors caractérısées à l'amidoschwartz et les glyco-protéines et polyosides par la réaction à l'acide périodique de SCHIFF adaptée par URIEL (17) à l'analyse immunoélectrophorétique.

\section{RÉSULTATS}

\section{A. - Composition du produit isolé.}

Le rendement de cette méthode d'extraction est d'environ 3 p. 100 par rapport au poids initial de bactéries sèches (lyophilisées).

La poudre blanche obtenue par lyophilisation est extrêmement pulvérulente ; on la remef facilement en solution dans l'eau, à moıns qu'elle n'ait subi des lyophilisations successives, ce qui altère grandement sa solubilité.

L'analyse des phosphates qu'elle contient, faite en deux temps, permet de séparer les phosphates acido-solubles, dosables directement sans hydrolyse ni minéralısation préalable (orthophosphates et esters phosphorylés très labiles tels que ribose-1-phosphates, etc..) des phosphates acidosolubles totaux.

La composition élémentaire de cet antigène purifié est résumée dans les, chiffres suivants qui sont des pourcentages en poids ef sontexprimés en phosphore minéral pour les phosphates:

Azole total. Phosphore Phosphore Protéine. A. R. N. O mn.* acidos. total.

$$
\text { 2,7 p. } 100 \quad 1,3 \text { p. } 100 \quad 3,6 \text { p. } 100 \quad 0 \text { p. } 100 \quad 0 \text { p. } 100
$$

L'absence de protéines et d'acide ribonucléique est confirmée par l'aspect du spectre dans I'ultraviolet d'une solution aqueuse de l'antigène (1 $\mathrm{mg} / \mathrm{ml}, \mathrm{pH} 7,48)$, comparé à celui du surnageant de la dernière centrifugation préparative (fig. 1.).

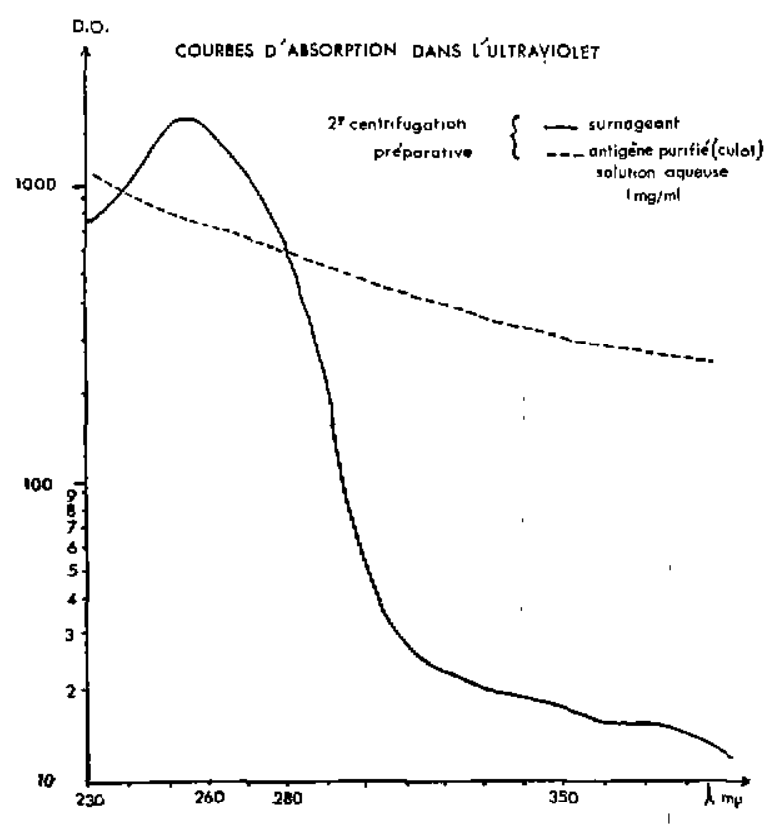

Fig. 1

* Phosphates dosables immédiatement avant l'hydrolyse ou la minéralisation. 
Pour l'acide ribonucléique on a :

$\frac{K 260}{K 310}=\frac{1190}{35}=48,28$ et $\frac{K 260}{K 230}=\frac{1690}{760}=2,22$ tandis que pour l'antigène : $\frac{K 260}{K 310}=1,68$ ef

$$
\frac{\mathrm{K} 260}{\mathrm{~K} 230}=0,67
$$

On peut d'abord conclure à l'absence de proteines contenant de la tyrosine, du tryptophane ou de la phényl-alanine, puisqu'aucun pic n'est visible à $280 \mathrm{~m} \mu$. En effet, on a pour la solution protéique témoin diluée 8 fois :

$$
\frac{K 280}{K 310}=26,85 \text { et } \frac{K 280}{K 250}=2,21 \text {, tandis que }
$$

pour l'antigène on $a: \frac{K 280}{K 310}=1,38$ ef $\frac{K 280}{K 250}$ $=0,75$

D'autre part, la réaction du biuret perfectionnée par ARDRY étant négative, on 'peut admettre, de façon définıtive, l'absence de protéines, absence qui, jointe à celle de l'acide ribonucléique, nous permet de penser que nous sommes en face d'un lipopolyoside purifié.

\section{B. - Analyse électrophorétique et immunoélectrophorétique}

Les électrophorèses sımples préliminaires nous ont confirmé l'absence de protéines décelables par nos méthodes d'analyse tandis que la caractérisation des glucides nous permettait de mesurer la mobilité du composé glucidique

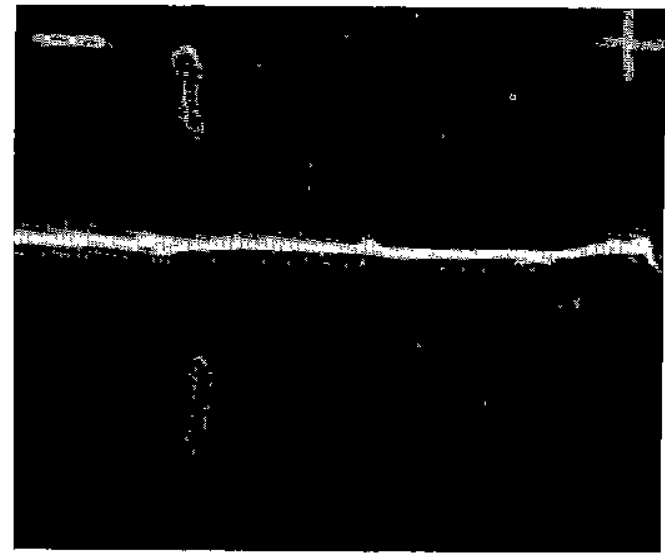

Fig. 2. - Analyse immunoélectrophorétique de principal de l'extrait relative à la sérum albumine humaine, soit : $+0,28$.

L'immunoélectrophorèse permet de différencier quatre arcs (fig. 2), dont deux sont très nets (lignes 1 ef 2); les deux autres sont plutôt des bandes de précipitation, d'apparition tardive, correspondant vraisemblablement à des fractions mineures de faible concentration (lignes 3 et 4 ).

\section{C. - Propriétés biologiques}

1) Chez le lapin (les animaux utilisés pèsent $2 \mathrm{~kg}$ ).

Le pouvoir pyrogène est très net; on observe régulièrement un pic thermique situé entre la deuxième heure et la quatrième heure qui suivent l'ınjection intraveineuse de lipopolyoside (voir fig. 3).

L'écart de température observé est d'au moins $2^{\circ} \mathrm{C}$ pour toutes les doses comprises entre $25 \mu \mathrm{g}$ et $0,05 \mu \mathrm{g}$. II est encore de 104 pour une dose de $0,005 \mu \mathrm{g}$.

On peut observer que des lapins immuns (ayant reçu la veille du sérum très riche en anticorps) font la même réponse fébrile, que le sérum soit homologue ou hétérologue par rapport au type de l'endotoxine injectée. Bien plus, Il s'y ajoute une réponse fébrile propre à l'injection de sérum de telle sorte que les lapins «protégés 》 font une réaction thermique plus élevée que celle des lapins normaux. Sans doute retouve-t-on là le pouvoir pyrogène des extraits leucocytaires que contiennent fréquemment les sérums.

L'action sur les leucocytes est également très nette, comme le montre la figure 4. La leucopénie

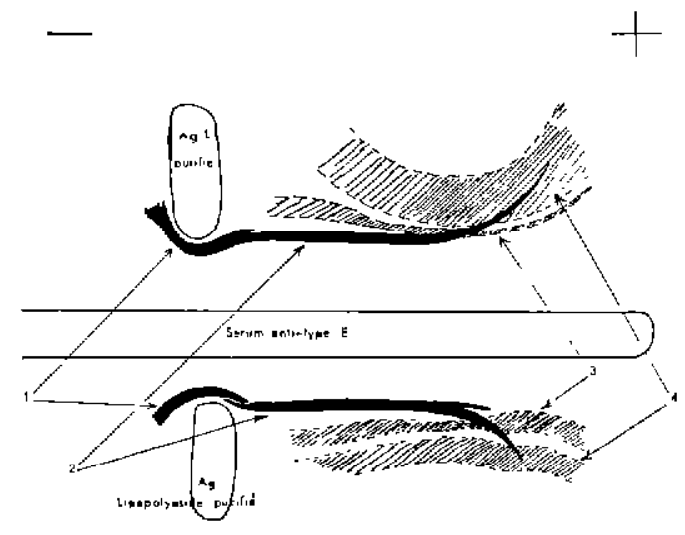

l'antigène lipopolyosidique purifié du type $E$. 
s'installe brutalement dans les minutes qui suivent l'injection et dure de 3 à 6 heures; elle est suivie d'une hyperleucocytose temporaire atteignant 25 à 30.000 leucocytes par $\mathrm{ml}$ en moyenne.

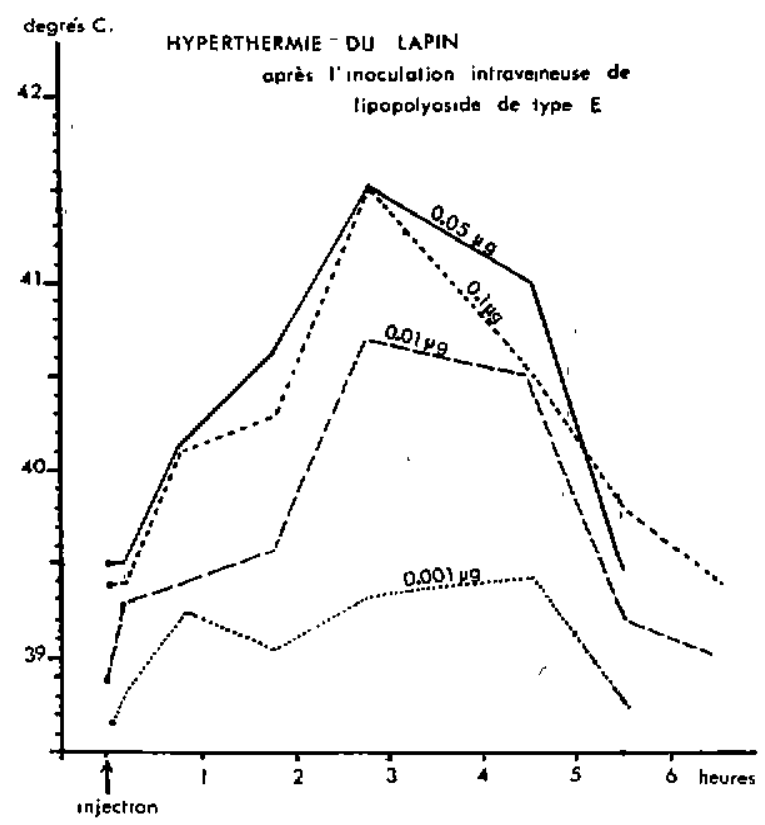

Fig. 3

Outre cette double action sur la température centrale et le nombre des leucocytes du sang, le lipopolyosıde provoque, par voie intraveineuse et pour des doses d'au moins $5 \mu \mathrm{g}$, un syndrome toxique constitué par :

- une crise respiratoire grave qui s'installe très vite après l'injection; elle peut aller de la

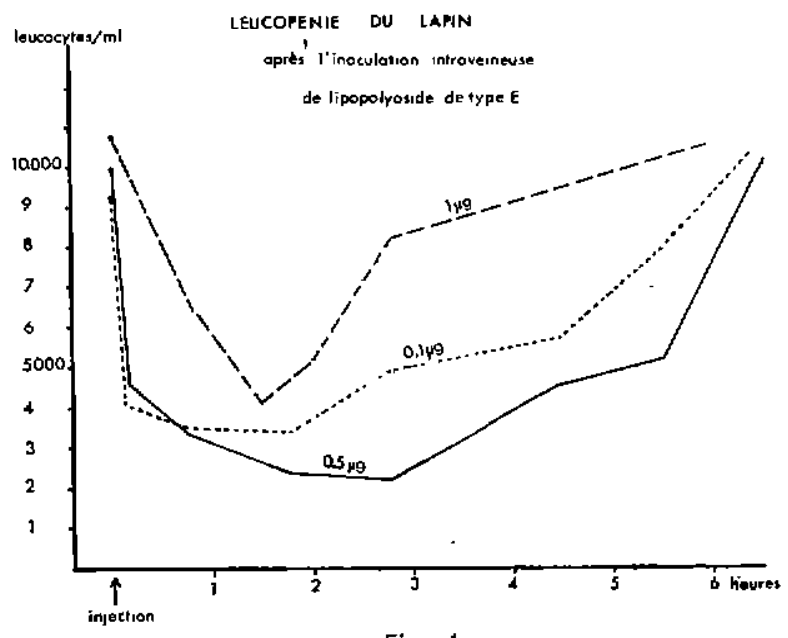

Fig. 4 simple polypnée à une dyspnée profonde avec tirage inspiratoire et discordance. Elle peut aussi être mortelle et l'issue fatale survenir en 1 ou 2 heures; noụs avons là les effets de la «toxine respiratoire » de Posteurella multocida qu'avaił signalée STAMATIN (16).

- des plaintes spontanées, des cris qui peuvent accompagner cette crise.

- de la diarrhée qui apparaît chez 50 p. 100 des lapins inoculés, souvent $15 \mathrm{mn}$ après l'ınjection.

— un état de prostration typhique fréquent, mais non obligatoire.

- des troubles vasculaires identiques à ceux observés classiquement avec les endotoxines des entérobactéries. Des phénomènes de vasoconstriction périphérique sont très visibles sur le réseau vasculaire de l'oreille du lapin; quelques minutes après l'injection intraveineuse, les vaisseaux superficiels subissent deux ou trois variations cycliques de diamètre, une vasodilatation succédant à une vaso-constriction ef chaque cycle durant 1 à $2 \mathrm{mn}$.

Ces troubles engendrent assez vite un état d'ischémı superficielle qui dure pendant toute l'évolution de l'intoxication et il devient très difficile de faire saigner la peau ou de prélever du sang à la veine marginale de l'oreille.

La dose sûrement mortelle pour le lapin peut être difficilement évaluée, car il semble qu'il y ait des différences très nettes de résıstance individuelle. Des animaux ont succombé très vite à une dose de $50 \mu \mathrm{g}$, d'autres ont résisté à une dose de $250 \mu \mathrm{g}$; il faut une dose d'au moins $500 \mu g$ pour que les lapins aient un risque important de mourir, mais des sujets y résistent encore.

Les lésıons macroscopiques observées sur les lapins tués par l'endotoxine pasteurellique sont essentiellement :

- des lésions très nettes d'emphysème du paumon et une congestion de la muqueuse de l'arbre trachéo-bronchique.

- une dégénérescence graisseuse marquée du foie.

- des pétéchies sur le thymus.

- une congestion discrète des plaques de Peyer.

- une congestion du tractus génital femelle (ovaires et cornes utérines surtout).

— une légère splénomégalie. 


\section{2) Chez lo souris:}

L'injection intrapéritonéale d'endotoxine entraîne de façon constante l'établissement d'une prostration accompagnée d'hypothermie et de diarrhée, ce qui reconstitue le tableau clinique observé chez les souris qui servent aux épreuves de séroprotection ef qui sont protégées insuffisamment ou pas du tout ; le retour à l'état normal survient au bout de 3 d̀ 4 jours.

L'appréciation de la dose mortelle pour la souris se heurte cux mêmes difficultés que chez le lapin, d'autant plus que celle-ci résiste fréquemment à des doses, considérables pour un tel animal, de $1 \mathrm{mg}$.

Les lésions les plus caractéristiques sont la dégénérescence graisseuse du foie ef la splénomégalie.

\section{3) Chez le zébu:}

Nous avons injecté le lipopolyoside par voie intraveineuse à six jeunes zébus (de race arabe du Tchad) âgés de un an à dix huif mois et pesant de 100 d̀ $140 \mathrm{~kg}$.

La préparation de lipopolyosıde qui nous a servi dans cette expérience n'était que peu purifiée et contenait encore de l'acide ribonucléique.

Les doses utilisées furent : $500 \mu \mathrm{g}, 1 \mathrm{mg}, 2 \mathrm{mg}$ ef $3 \mathrm{mg}$.

D'une façon générale, les réactions n'ont pas eu l'intensité que l'on espérait; la réponse la plus nette a été constituée par la leucopénie consécutive à l'injection intraveineuse (voir le tableau no 1).

Plus de réaction d'hyperthermie marquée: pour une dose de $500 \mu \mathrm{g}$, l'écart de tempérałure a atteint au maximum 102 et encore faudrait-il tenir comple de la montée habituelle de la température centrale pendant les heures chaudes de la journée.

Pour les doses de 1,2 et $3 \mathrm{mg}$, l'effet le plus inattendu fut une réaction d'hypothermie (voir fig. 5) qui coïncidait avec un syndrome toxique net.

La nature de la réponse thermique dépendraitelle de la dose d'endotoxine injectée ? Nous avons fait trop peu d'essais pour pouvoir l'affirmer.

Les animaux se tenaient prostrés, le mufle au ras du sol: polypnée, ptyalisme abondant, émissions fréquentes d'excréments ramollis, plainte spontanée expiratoire constituaient les signes les plus nets.

Tout rentra dans l'ordre en quelques' heures et le lendemain les animaux étaient parfaitement normaux.

\section{D. - Propriétés antigéniques}

\section{1. - In vitro:}

a) Hémagglutination indirecte :

Cet antigène purifié possède une grande affinité pour la surface des hématies humaines $O$ et des hématies de mouton, qu'elles soient fraîches ou formolées et il devient donc facıle d'effectuer des tests d'hémagglutination indirecte, réactions dont la spécificité devient excellente.

CARTER (6) vient d'ailleurs de montrer l'intérêt que pouvalt avoir l'emploi des lıpopo-

Tableau no I

Chute du nombre des leucocytes après l'injection intraveineuse

de lipopolyoside E chez les jeunes zébus.

\section{No des animaux}

\begin{tabular}{|c|c|c|c|c|c|c|}
\hline & 697 & 699 & 969 & $\mathrm{SN}$ & 698 & 889 \\
\hline Dose de 11popolyoside E & $500 \mathrm{\mu g}$ & $500 \mathrm{\mu g}$ & $1 \mathrm{mg}$ & 1 mg & $2 \mathrm{mg}$ & $3 \mathrm{mg}$ \\
\hline \multicolumn{7}{|l|}{ Leucocytes / ml } \\
\hline 1) nombre initial : & 12000 & 12800 & 12000 & 5600 & 9000 & 9400 \\
\hline 2)nombre minimum observé ; & 4000 & 3200 & 1200 & 1000 & 3400 & 2800 \\
\hline
\end{tabular}




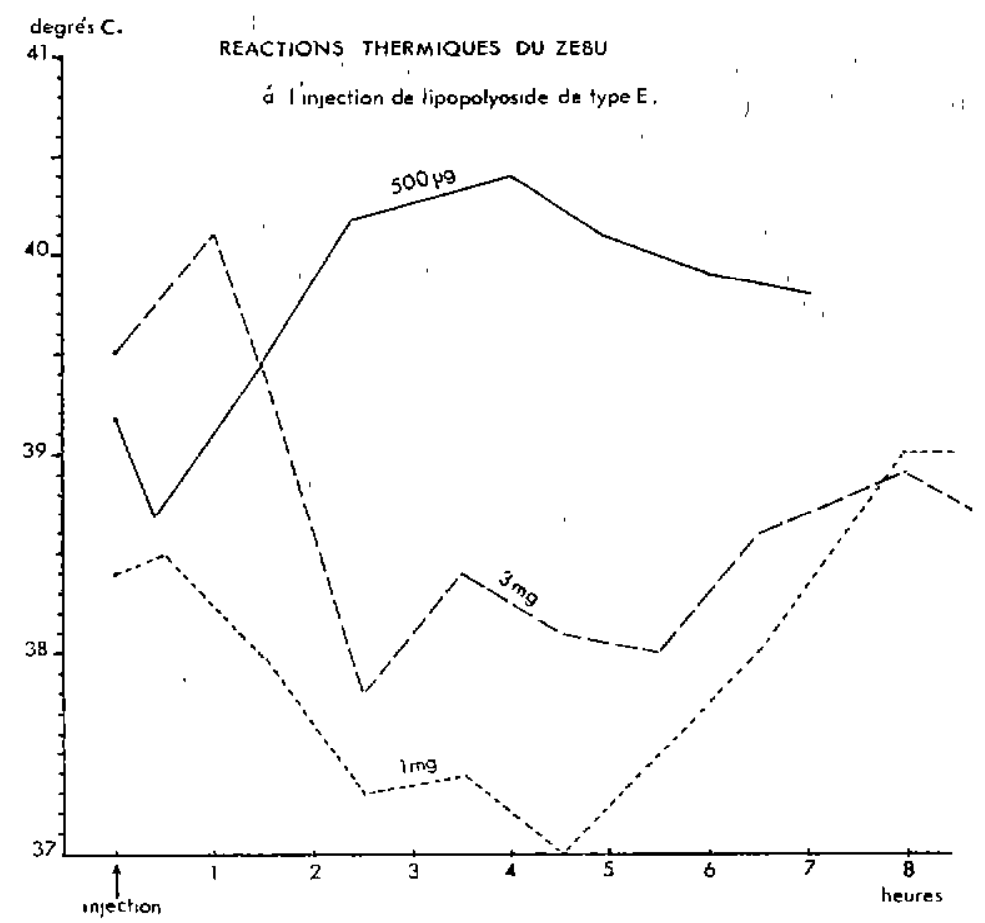

Fig. 5

lyosides spécifiques des types $A, B$ et $D$ de Pasteurella multocida dans l'étude immunologique des pasteurelloses; nos expériences confirment ces travaux et les complètent avec le type $E$ africain.

Les tableaux suivants ( $n^{\circ} \|$ et $n^{\circ}$ III) montrent les quantités de lipopolyosıde nécessaires pour sensibiliser correctement la surface des hématies et cela de façon comparative pour le type B et le type $E$.

On y voit que, pour les deux antigènes puriflés, des quantités de 2,5 à $10 \mu g$ sont suffisantes pour saturer efficacement la surface des hématies contenues dans $0,1 \mathrm{ml}$ de culot normal de centrifugation, soit la quantité d'hématies permettant d'obtenir $10 \mathrm{ml}$ de suspension d̀'1 pour cent.

Tableau $n^{\circ}$ II

Réection d'hémagglutination pasaive avec le lipopolyoside purifié de type E (souche P 13)

\begin{tabular}{|c|c|c|c|c|c|c|c|c|c|c|}
\hline \multicolumn{2}{|c|}{ Quantités de } & \multicolumn{8}{|c|}{ Dilutions du sórum } & \multirow[b]{2}{*}{$1 / 20480$} \\
\hline lipopol & yoside*: & $1 / 80$ & $1 / 160$ & $1 / 320$ & $1 / 640$ & $1 / 1280$ & $1 / 2560$ & $1 / 5120$ & $1 / 10240$ & \\
\hline 50 & $\mu 8$ & + & + & + & + & + & + & + & - & - \\
\hline 25 & 18 & + & + & + & + & + & + & + & - & - \\
\hline 10 & $\mu g$ & + & + & + & + & + & + & + & + & - \\
\hline 5 & $\mu \mathrm{g}$ & + & + & + & + & + & + & + & + & \pm \\
\hline 2,5 & $\mu g$ & + & + & + & + & + & + & + & + & - \\
\hline 1 & $\mu 8$ & - & - & - & - & - & - & - & - & - \\
\hline
\end{tabular}

* pour senslbiliser $0,1 \mathrm{ml}$ de culot d'hématies qui seront utilisées en suspension à 1 p.100 
Ces quantités d'antigène sont assez inférieures à celles qu'indiquent les expériences de CARTER (6) ; sans doute faut-il rechercher la raison de cette différence dans l'inégalité des degrés de purification.

La réaction d'hémagglutination passive permet de mettre en évidence, sans contestation possible, la nan-identıté antigénique des deux lipopolyosides spécifiques $B$ et $E$ (tableau no IV). n'est pas totale dans le cas de l'antisérum E pour une même masse d'antigène, ce qui peut s'expliquer soit par une faible réaction croisée, soit par une saturation incomplète des anticorps (il aurait fallu plus de $100 \mu \mathrm{g}$ de lipopolyoside E).

Quoiqu'il en solt, les épreuves d'hémagglutınation normale ou inhibée montrent qu'il existe un antigène spécifique du type $B$ et un autre spécifique du type $\mathrm{E}$.

Tableau No III

Réaction d'hémagglutination passive avec le lipopolyoside

purzfié de type $\mathrm{B}$ (souche Insein)

\begin{tabular}{|c|c|c|c|c|c|c|c|c|}
\hline $\begin{array}{l}\text { Quantltés de } \\
\text { lupopolyoside * }\end{array}$ & \multicolumn{8}{|c|}{ Dilutions du sárum } \\
\hline $50 \mu g$ & + & + & + & + & + & + & - & - \\
\hline $25 \mu g$ & + & + & $r$ & + & + & + & - & - \\
\hline $10 \mu 5$ & + & + & + & + & + & \pm & - & - \\
\hline $5 \mu g$ & + & + & + & + & r & $\doteq$ & - & - \\
\hline $2,5 \mu \mathrm{g}$ & + & t & 2 & + & \pm & - & - & - \\
\hline $1 \mu g$ & r & + & \pm & - & - & - & - & - \\
\hline $0,5 \mathrm{~kg}$ & - & - & - & - & - & $\rightarrow$ & - & - \\
\hline
\end{tabular}

* pour senslbjliser 0,1 ml de culot d'hématies quz seront utılisées en suspenszon à 1 p.100

II existe cependant une réaction croisée positive au $1 / 20$ (sérum $B$ sur antigène $E$ ), donc assez faible, que nous avions déjà signalée dans nos premières observations.

Le fableau $n^{\circ} \mathrm{V}$ résume les expériences d'inhibition homologue ou croisée de l'hémagglutination; on observe qu'avec $100 \mu \mathrm{g}$ d'antigène purifié on peut inhiber complètement l'activité de $0,4 \mathrm{ml}$ d'antisérum $B$ au 1/10. L'inhıbition b) Diffusion-précipitation en milieu gélifié : Cette méthode révèle, de façon tout aussi netfe, l'individualité du type $E$. Une ligne de précipitation très dense et brillante apparaît rapidement en milieu gélosé, lorsqu'un sérum

Tabiean No IV

Réactions d'hémagglutination crolséo

\begin{tabular}{|c|c|c|c|c|c|c|c|c|c|c|c|}
\hline \multirow[b]{2}{*}{ Sérums } & \multirow[b]{2}{*}{$1 / 10$} & \multicolumn{9}{|c|}{ 10) Hématies sensibilzsées par le lipopolyoside B. } & \multirow[b]{2}{*}{$1 / 10240$} \\
\hline & & $1 / 20$ & $1 / 40$ & $1 / 80$ & $i / 160$ & $1 / 320$ & $1 / 640$ & $1 / 1280$ & $1 / 2560$ & $1 / 5120$ & \\
\hline Anti-B & + & + & + & + & + & + & + & + & + & + & - \\
\hline \multirow[t]{2}{*}{ Anti-E } & - & - & - & - & - & - & - & - & - & - & - \\
\hline & \multicolumn{11}{|c|}{ 20) Hématies sensibilisées par le lipopolyoside E. } \\
\hline Anti-B & + & + & - & - & - & - & - & - & - & - & - \\
\hline Anti-E & + & + & + & + & + & + & + & + & + & + & - \\
\hline & & & & & & 12 & & & & & \\
\hline
\end{tabular}


Tableau $\mathbf{n}^{\circ} \mathrm{V}$

Réactions d'inhibition de l'hémagglutination

\begin{tabular}{|c|c|c|c|c|c|c|c|c|c|c|c|c|}
\hline \multirow[t]{2}{*}{ Sérums } & $\begin{array}{c}\text { Eláment } \\
\text { inhibiteur }\end{array}$ & $\begin{array}{l}\text { Bématies } 0 \\
\text { sensibilisées } \\
\text { par }\end{array}$ & \multicolumn{10}{|c|}{$\begin{array}{l}\text { Dilutions des sérums at rósultats : } \\
1 / 10 \quad 1 / 20 \quad 1 / 40 \quad 1 / 80 \quad 1 / 160 \quad 1 / 320 \quad 1 / 640 \quad 1 / 1280 \quad 1\end{array}$} \\
\hline & $\begin{array}{c}\text { Sérum } \\
\text { physiologique }\end{array}$ & & 4 & 4 & 4 & 4 & 4 & 4 & 4 & 4 & 2 & 0 \\
\hline \multirow[t]{3}{*}{ Anti-B } & $\begin{array}{c}\text { Lipopolyoside E } \\
100 \mu 8\end{array}$ & $\underset{B}{A n t i g e ̀ n e ~}$ & 4 & 4 & 4 & 4 & 4 & 4 & 4 & 3 & 0 & 0 \\
\hline & $\begin{array}{c}\text { Lipopolyoside B } \\
100 \mathrm{\mu g}\end{array}$ & & 0 & 0 & 0 & 0 & 0 & 0 & 0 & 0 & 0 & 0 \\
\hline & $\begin{array}{l}\text { Sérum } \\
\text { physiologique }\end{array}$ & & 4 & 4 & 4 & 4 & 4 & 4 & 2 & 0 & 0 & 0 \\
\hline \multirow[t]{2}{*}{ Anti-E } & $\begin{array}{c}\text { Lipopolyoside B } \\
100 \mathrm{ps}\end{array}$ & $\underset{E}{\operatorname{Antigène}}$ & 4 & 4 & 4 & 4 & 4 & 4 & 0 & 0 & 0 & 0 \\
\hline & $\begin{array}{c}\text { Lipopolyoside E } \\
100 \mu \mathrm{g}\end{array}$ & & 3 & 0 & 0 & 0 & 0 & 0 & 0 & 0 & 0 & 0 \\
\hline
\end{tabular}

NB. : Le réaction utilise les volumes suivants : - 0,4 ml de chaque dilution de sérum.

- $0,1 \mathrm{ml}$ de solution de lipopolyoside à $1 \mathrm{mg} / \mathrm{ml}$, (soit $100 \mathrm{\mu g}$ ) ou de sérum physiologique.

- 0,4 ml de suspension à 1 p.100 d'hémeties aenslbilisées.

- Volume total par tube : $0,9 \mathrm{ml}$.

Los dilutions de sérum restent 2 helures à $37^{\circ}$ en contact avec lo lipopolyoside inhibiteur (ou le sérum physiologique) avant de recerolr la suspension d'hématies.

anti-E est opposé au lipopolyoside E : il s'agit là d'un phénomène spécifique. Aucune ligne n'apparaît lorsque ce même sérum est opposé aux lipopolyosides des types $A$ et $D$ (voir la fig. 6) ; par contre, une ligne flave, d'apparition très lente, décalée par rapport à l'arc spécifique $E$ apparaît entre le réservoir du sérum anti-E et celui de l'antigène purifié de type $B$. Cette dernière ligne disparaît régulièrement lorsque ce sérum a été absorbé par des bactéries entières ou par le lipopolyoside de type B.

Il semble donc qu'un sérum anti-E possède à la fois des anticorps spécifiques $E$, en majeure partie, et des anticorps $B$ en petıte quantité ; ce serait là le reflet de la composition antigénique des lipopolyosides des bactéries de type $\mathrm{E}$.

$\mathrm{Ce}$ serait aussi l'explication des réactions croisées aux basses dilutions de sérum dans les épreuves d'hémagglutination.
2. - In vivo:

Le lipopolyoside $E$ injecté à doses répétées à des souris ne provoque, semble-t-il, l'élaboration d'aucun anticorps protecteur: Des souris qui ont reçu 6 injections sous-cutanées de $50 \mu \mathrm{g}$ d'antigène du type $B$ ou $E$ ne sont aucunement protégées contre 1.000 doses sûrement mortelles de Pasteurella multocida type $B$ ou $E$ (épreuve homologue ou croisée), ainsi qu'en témoigne le tableau $n^{\circ} \mathrm{VI}$.

On pourrait en conclure que cet antigène $n$ 'a aucun rôle dans l'élaboration des anticorps protecteurs; mais la méthode de WESTPHAL ne nous permet sans doute d'obtenir qu'un « haptène » et non l'antigène complet: haptène + protéine.

Nous avons essayé d'absorber un sérum anti-E par le lipopolyoside spécifique afin de voir si le pouvoir protecteur de ce sérum s'en trouvaif modifié. 

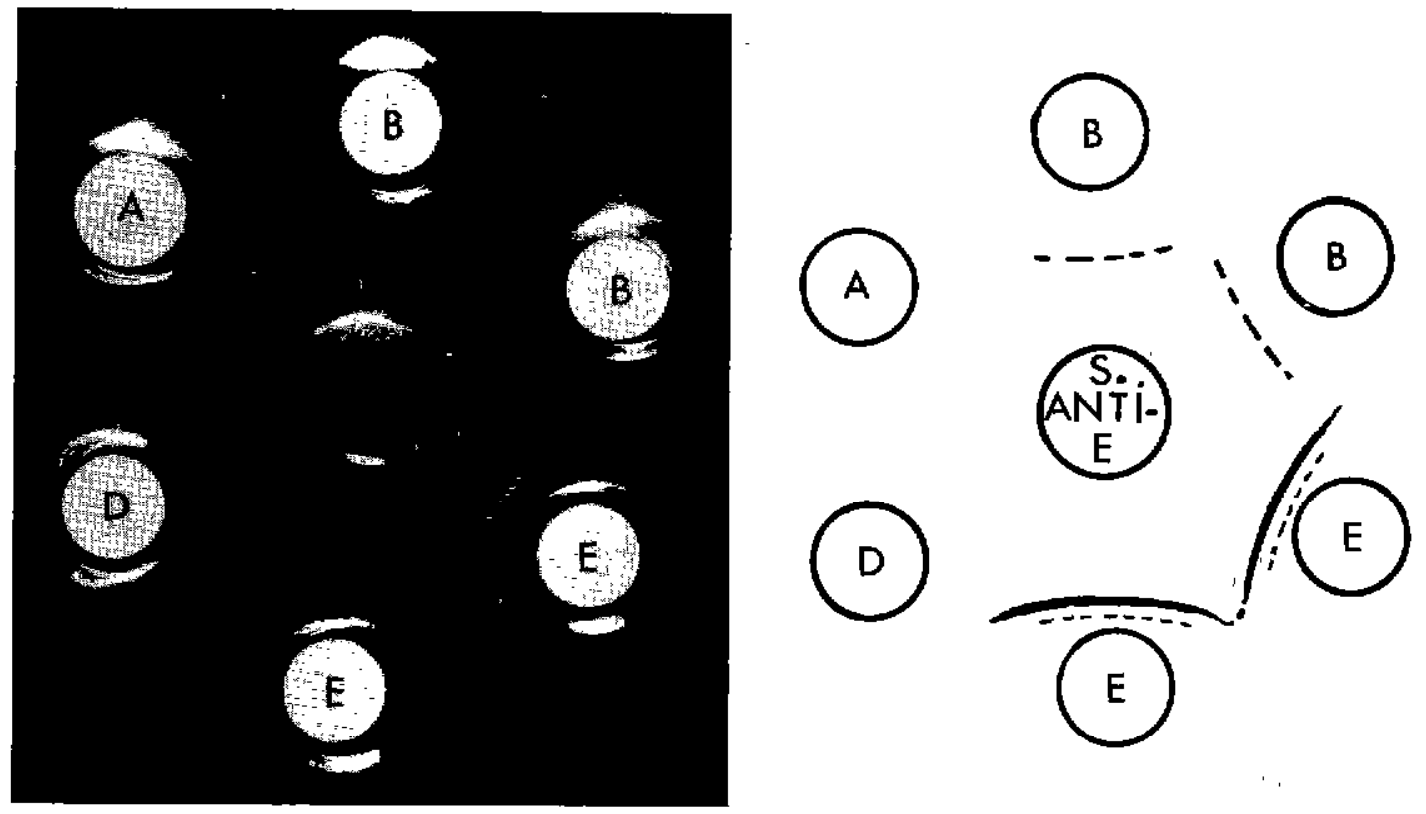

Fig, 6. - Précipitation en gélose : sérum anti-E (souche $P_{7}$ ) et lipopolyosides des types A (souche 2077), B (souches insein et 853), $D$ (souche 1554) ef $E$ (souche $P_{13}$ ).

La photo montre l'arc spécifique du type $E$; le schéma indique en outre, en trait pointilié, la ligne de précıpitation caractéristique de la fraction lipopolyosidique commune aux types $B$ el $E$, non visible sur la photo.

En trois absorptions successives exécutées à la température de $4^{\circ}$, une quontité globale d'environ $10 \mathrm{mg}$ de lipopolyoside est nécessaire pour supprimer totalement l'activité agglutinante et précipitante de $4 \mathrm{ml}$ d'un bon sérum anti-E (hémagglutınation positive au 1/2560, protection de la souris à la dose de $0,05 \mathrm{ml}$ contre au moins 10.000 D. S. M.).

Le pouvoir protecteur se trouve fortement réduit : 50 p. 100 des souris seulement survivent à une dose de 1.000 D. S. M. après avoir reçu $0,1 \mathrm{ml}$ de ce sérum absorbé.

L'absorption des anticorps protecteurs n'est donc que partielle et il est vraisemblable que d'autres antigènes bactériens interviennent dans l'éloboration de l'immunité ; mais il est permis de penser que l'antigène lipopolyosidique «complet»tel qu'il existe dans la bactérie, lié à des éléments protéiques, provoque la formation in vivo d'une partie au moins des anticorps protecteurs et peut donc se classer parmi les antigènes vaccinants.

Adsorbé sur des hématies de mouton et injecté au lapin par voie intraveineuse (8 injections à 3 jours d'intervalle de $1 \mathrm{ml}$ d'une suspension à 2 p. 100 d'hématies sensibilisées), ce lipopolyo- side provoque la formation d'agglutinines à un titre qui s'est élevé dans nos essais, jusqu'au $1 / 5120$ (en hémagglutination passive) ; le sérum des lapins ainsi immunisés contient "également des précipitines, mais à un titre beaucoup plus faible.

II a aussi un pouvoir protecteur très net; à la dose de $0,1 \mathrm{ml}$ en injection sous-cutanée, it protège la souris contre 10.000 doses sûrement mortelles'd'une souche de Pasteurella 'multocida de même type; il s'agit donc bien d'un antigène vaccinant.

Cette protection est très spécifique et ne vaut que pour le sérotype $E$; aucune 'souris ainsi prớégée $(0,1 \mathrm{ml}$ de sérum 'injecté la veille de l'épreuve) ne résiste à une infection de 100 D. S. M. par le sérotype B*.

Ces résultats s'accordent très bien avec le parallélisme constamment observé dans l'espèce Pasteurella multocida entre la spécificité sérologique et la spécificité immunologique.

* Dans ces expériences, la dose sûrement morteile pour la souris est de $0,1 \mathrm{ml}$ ì la dilution $10^{-8}$ d'une hémoculture de 6 heures, pour les souches B (Insein, 215 Roberts) et les souches $E\left(P_{7}, P_{19}\right)$. 
Tableau no VI

Essai d'imrunisation de la souris par les lipopolyosides $B$ et E.

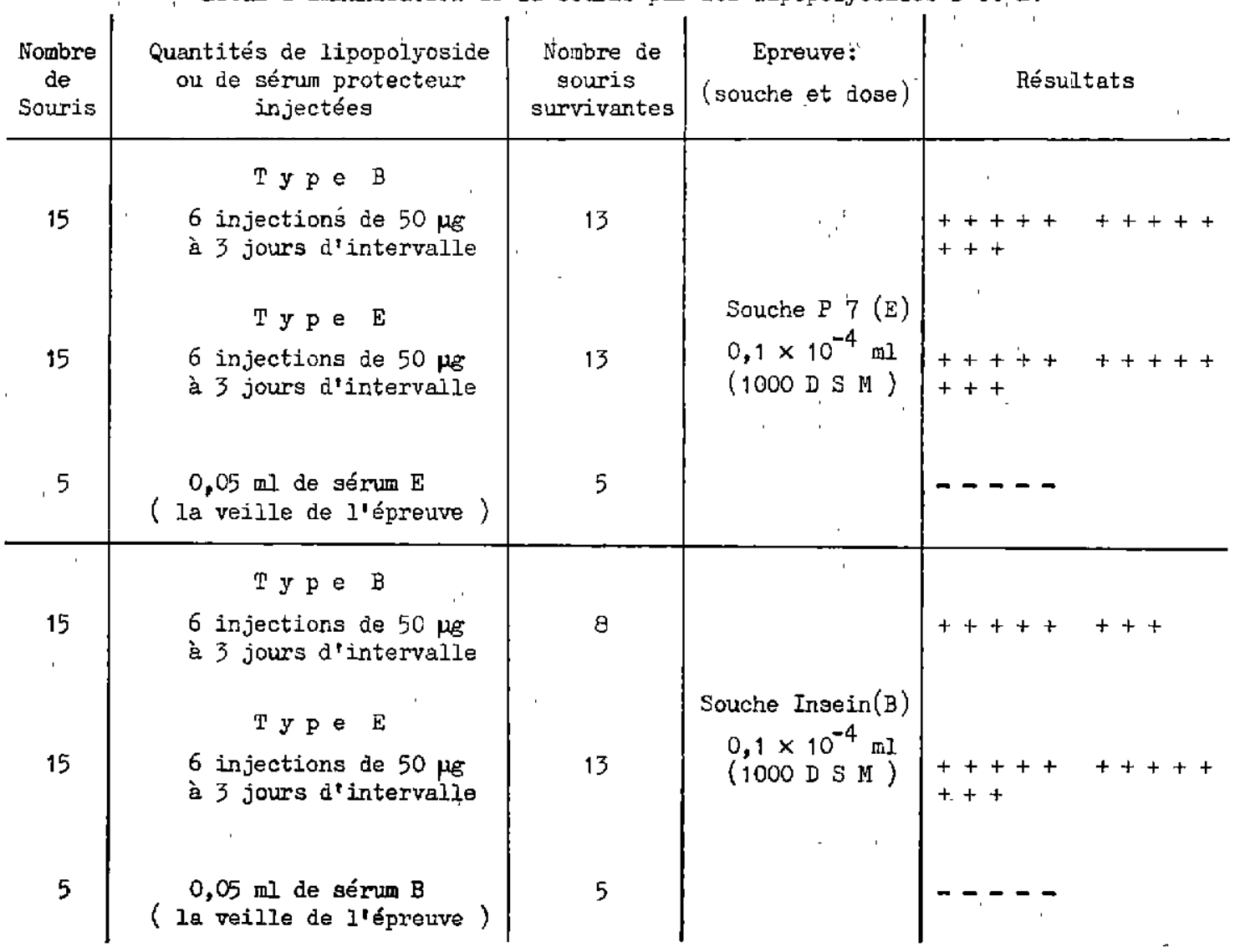

NB. La survie est indiquée par le signe - , la mort par le aigne +

\section{COMMENTAIRES}

Sur le plan technique, il est difficile, même èn opérant de façon rigoureusement identique, d'obtenir par la méthode de WESTPHAL des lots d'antigène strictement comparables en ce qui concerne d'une part leur composition chimique élémentaire, d'autre part leur activité sérologique qui, si elle reste qualitativement la même, varie souvent quantitativement de façon assez importante d'un lot à l'autre, et surtout si, sans changer de type sérologique, on change de souche.

Un fait est certain : le lot de lipopolyoside purifié qui s'est révélé le plus actif sérologiquement a été préparé avec une souche de $P$. multocida isolée un mois avant et n'ayant subi que deux subcultures; nos autres souches, bien que conservées avec beaucoup de précautions, nous ont donné des résultats satisfaisants mais nettement moins bons.

Nos observations, quant au pouvoir toxique de l'antigène purifié, concordent exactement avec celles de BAIN et KNOX (2) au sujet des lipopolyosides du type $B$; nous avons constaté, à mesure que progressait notre technique de préparation et qu'étaient purifiés nos lots de lipopolyosides, que le pouvoir toxique semblait diminuer, en même temps que diminuait le pourcentage en azote. C'est la fraction lipidique qui, en principe, est responsable de la toxicité ; mais ne pourrait-il s'y ajouter le rôle toxique d'un autre élément (azoté ou protéique), lequel aurait existé dans nos préparations en tant d'impureté ?

Sur le plan sérologique, il n'est guère besoin 
de commenter l'intérêt que l'on peut avoir à disposer d'un antigène purifié, extrêmement spécifique et titrable de manière très précise; il est sûr que l'immunologie des pasteurelloses et la sérotypie des souches en profiteront largement.

II semble que l'on puisse admettre que le sérotype $\mathrm{E}$ de Pasteurella multocida est caractérisé par un groupement d'antigènes lipopolyosidıques comprenanf, d'une part et en majeure partie, un antigène spécifique déterminant le type $E$, d'autre part et en faible quantité une fraction commune avec les lipopolyosides de type B.

Une structure antigénique parallèle se rencontrerait dans les lipopolyosides du type B : d'abord, la fraction spécifique $B$, ensuite la fraction commune, celle-ci beaucoup moins importante que la première; c'est ce qu'il semble ressortir de nos essais d'analyse en milieu gélifié, analyse que nous nous proposons de pousser plus à fond.

\section{CONCLUSION}

La méthode de WESTPHAL permet d'isoler de Pasteurella multocida type $E$ un antigène lipopolyosidique ayant les propriétés générales des endotoxines des germes gram-négatifs.

Sa composition élémentaire, ses caractères de toxicité et ses propriétés sérologiques sont absolument parallèles à ceux que présente le lipopolyoside du type $B$ de Pasteurella multocida.

Mais les techniques sérologiques montrent que cet antigène est qualitativement différent de celui du type $B$; la réalité du type $E$ africain s'en trouve donc confirmée, le type sérologique étant, d'une façon commune, déterminé chez les gram-négatifs par la nature des antigènes lipopolyosidiques.

Comme les lipopolyosides spécifiques des autres types de Pasteurella multocida l'endotoxine $E$ est l'antigène responsable du phénomène de l'hémagglutination passive. II possède, in vivo, un pouvoir antigénique certain ; adsorbé sur des hématies injectées au lapin, il permet d'obtenir un sérum agglutinant, précipitant ef protecteur pour la souris.

Il reste à approfondir, par voie analytique, les différences de composition chimique qui existent entre les lipopolyosides $B$ et $E$ et que révèle, pour le moment, la sérologie seule; c'est ce que nous nous proposons dans un travail ultérieur.

\section{Instituf d'Elevage ef de Médecine vétérinaire des Pays tropicoux. \\ Laboratoires de microbılogie et de chimie biologique d'Alfort. Laboratoire de Farcha-Fort-Lamy.}

\section{SUMMARY}

\section{Glycolipoid antigen of P. multocida. Type E.}

A glycolipoid antigen has been isolated from P. multocida type $E$ by Westphal's method. It has the general properties of endotoxins from gram-negative organısms.

Its elementary composition, toxic characters and serological properties are absolutely parallel to type B lipopolysaccharide of the same organism.

Serologic techniques show however that the type $E$ antigen is qualitatively different to type $B$. The identity of a type $E$ antigen of African origin is thus confirmed.

As with the specific glycolipoids of other types of $P$. multocida, endotoxin $E$ is the antigen responsible for the phenomenon of passive haemagglutination. In vivo it has a definite antigenic property and when adsorbed to R. B. C. and injected into rabbits, it produces a serum which agglutinates and precipitates and protects mice.

For the time being only the serological differences are known between these two antigens, $B$ and $E$, and it remains to detect by analysis the differential chemical compositions. This it is proposed to work on. 


\section{RESUMEN}

\section{Antigeno lipopoliosidico de pasteurella multocida, tipo $\mathrm{E}$}

El método de Westphal permite asilar de «Pasteurella multocida » tipo E un antigeno lipopoliosídico que posee las propiedades generales de las endotoxinas de los gérmenes gram-negatıvos.

Su composición elemental, sus características de toxicidad y sus propiedades serológicas son ábsolutamente paralelas a aquellas que presenta el lipopoliosido del tipo B de « Pasteurella multocida 》.

Pero, las técnicas serológicas demuestran que este antigeno es cualitativamente diferente de aquel del tipo B ; la realidad del tıpo E africano se encuentra, pues, confirmada, ya que el tipo serológico queda, de forma general, determinado en el caso de los gram-negativos por el propio género de los antigenos lipopoliosídicos.

Como los lipopoliosídicos específicos de los demás tipos de «Pasteurella multocida», la endotoxina $E$ constituye el antigeno responsable de la hemaglutinacián pasiva y posee, in vivo, un poder antigénico indiscutible. En absorción sobre hematies inyectadas al conejo, permıte obtener un suero aglutinante, precipitante $y$ protector para el ratón.

Quedan aún por profundizar, por vía analítica, las diferencias de composición química que existen entre los lipopoliosidos B y E y que pone de manifiesto, por el momento, la serología únicamente. Esto es to que nos proponemos realizar en un trabajo ulterior.

\section{BIBLIOGRAPHIE}

1. $\operatorname{ARDRY}\left(R_{1}\right)$. - Le dosage des protéines par la réaction du biuret, détermination d'un coefficient spécifique d'absorption. Ann. Biol. clinique, 1960, 18 (3-4) : 214-22.

2. BAIN (R. V. S.) ef KNOX (K.W.). - The antigens of Pasfeurello multocida type I. Il. Lipopolysaccharides. Immunology, 1961, 4 (2) : 122-9.

3. CARTER (G. R.). - - Studies on Pasteurella multocida 1. A. haemagglutination test for the identification of serological types. Amer. J. Vef. Res., 1955, $16: 481-4$.

4. CARTER (G. R.). - A new serological type of Pasteurello multocida from Central Africa. Vet. Rec., 1961, 73 (42) : 1052.

5. CARTER (G. R.) et ANNAU (E.). - - Isolation of capsular polysaccharides from colonial variants of Pasteurella multocida. Amer. J. Vet. Res., 1953, 14: 475-8.

6. CARTER (G. R.) et RAPPAY (D. E.). - A haemagglutination test employing specific lipopolysaccharides for the detection and measurement of Pasteurella antibodies to « Pasteurella multocida ». Brit. Vet. J., 1963, 119 (2) : 73-7.

7. DELSAL (J. L.) et MANHOURI (H.), - Etude comparative des dosages colorimétriques du phosphore. Recherche d'une méthode de haute sensibilité applicable au dosage du phosphore organique dans les spots après chromatographie. Bull. Soc. Chim. Biol., 1955, 37 (9-10) : 1041-7.

8. GRABAR (P.) et BURTIN (P.). - Analyse immuno-électrophorétique. Ses applications aux liquides biologiques humains. 1960, Masson et Cie, éditeurs, Paris.

9. MAC LENNAN (A. P.) et RONDLE (C. J. M.). - Pasteurella septica : the occurrence of type specific polysaccharides containing aldoheptose sugars. Noture (London), 1957, 180 (4594) : 1045-6.

10. PERREAU (P.). - La culture dense de Pasteurella mulfocida, méthode de choix pour la production du vaccin contre la pasteurellose bovine. Rev. Elev. méd. Vét. Pays trop., 1961, 14 (2) : 133-40.

11. PERREAU (P.). - Contribution à l'étude immunologique de Posteurella multocida. Existence et importance d'un nouveau type, agent de la septicémie hémorragique des bovidés africains. Rev. Elev. Méd. vét. Pays trop., 1961, 14 (3) : 245-56.

12. PIROSKY (1.) - Sur l'antigène glucidolipidique des Pasteurello. Compt, rend. Soc. de Biol., 1938, 127 : 98-100. 
13. PIROSKY (I.). - Sur l'existence, chez les variants Smooth et Rough d'une souche de Pasteurella aviseptico, de deux antigènes glucido-lipidiques sérologiquement distincts. Compt, rend. Soc de Biol., 1938, I28: 346-7.

14. PIROSKY (I.). - - Sur les propriétés immunisantes, antitoxiques et anti-infectieuses de l'antigène glucido-lipidique de « Pasteurella aviseptica ». Compt, rend. Soc. de Biol., 1938, 127 : 966-9.

15. SCHEIDEGGER (J. J.). - Inter. Ach. Allergy Appl. Immunol., 1955, $7: 103$.

16. STAMATIN (N.), SERBANESCU (C.) et VLADEANU $\left(M_{1}\right)$. - L'activité pathogène de la toxine des Pasteurella pour quelques espèces animales. Ann. Inst. Pasteur, 1949, $76: 84$.

17. URIEL (J.) et GRABAR (P.). - Emploi de colorants dans l'analyse immunoélectrophorétique en milieu gélifié. Ann. Inst. Pasteur, 1956, 90 (4) : 427-40.

18. WESTPHAL (O.). - Pyrogens. Polysaccharides in biology. 1957, p. 115-220, édité par Josiah Macy Jr. Foundation, New-York, N. Y.

19. WESTPHAL (O.). - Récentes recherches sur la chimie et la biologie des endotoxines des bactéries à gram-négatif. Ann. Inst. Posteur, 1960, 98 (6) : 789-813. 